\title{
Within patient comparison of His-bundle pacing, right ventricular pacing and right ventricular pacing avoidance algorithms in patients with PR prolongation: Acute haemodynamic study
}

\author{
Daniel Keene ${ }^{1}$, Matthew Shun-Shin ${ }^{1}$, Ahran Arnold ${ }^{1}$, Katherine March ${ }^{1}$, Norman Qureshi ${ }^{2}$, \\ Fu Siong $\mathrm{Ng}^{3}$, Mark Tanner ${ }^{2}$, Nicholas Linton ${ }^{2}$, Phang Lim ${ }^{2}$, David Lefroy ${ }^{4}$, Prapa \\ Kanagaratnam ${ }^{2}$, Nicholas Peters ${ }^{5}$, Darrel Francis ${ }^{6}$, and Zachary Whinnett ${ }^{3}$ \\ ${ }^{1}$ Imperial College \\ ${ }^{2}$ Imperial College Healthcare NHS Trust \\ ${ }^{3}$ Imperial College London \\ ${ }^{4}$ Imperial college Healthcare \\ ${ }^{5}$ St. Mary's Hospital \\ ${ }^{6}$ St Mary's Hospital and Imperial College London
}

June 1, 2020

\begin{abstract}
Aims: A prolonged PR interval may adversely affect ventricular filling and therefore cardiac function. AV delay can be corrected using right-ventricular-pacing (RVP) but this induces ventricular dyssynchrony, itself harmful. Therefore, in intermittent heartblock, pacing-avoidance algorithms are often implemented. We tested His-bundle pacing (HBP) as an alternative. Methods: Out-patients with a long PR interval $(>200 \mathrm{~ms})$ and intermittent need for ventricular pacing were recruited. We measured within patient differences in high-precision haemodynamics between AV-optimized RVP, and HBP, as well as a pacing-avoidance algorithm [Managed Ventricular Pacing (MVP)]. Results We recruited 18 patients. Mean left ventricular ejection fraction was $44.3 \pm 9 \%$. Mean intrinsic PR interval was $266 \pm 42 \mathrm{~ms}$ and QRS duration was $123 \pm 29 \mathrm{~ms}$. RVP lengthened QRS duration(+54 ms, $95 \%$ CI 42 to $67 \mathrm{~ms}, \mathrm{p}<0.0001)$ whilst HBP delivered a shorter QRS duration than RVP(-56 ms, $95 \% \mathrm{CI}-67$ to $-46 \mathrm{~ms}, \mathrm{p}<0.0001)$. HBP did not increase QRS duration(-2ms $95 \%$ CI -8 to $13 \mathrm{~ms}, \mathrm{p}=0.6)$. HBP improved acute systolic blood pressure by mean of 5.0 $\mathrm{mmHg}(95 \% \mathrm{CI} 2.8$ to $7.1 \mathrm{mmHg}, \mathrm{p}<0.0001)$ compared to RVP and by $3.5 \mathrm{mmHg}(95 \% \mathrm{CI} 1.9$ to $5.0 \mathrm{mmHg}, \mathrm{p}=0.0002)$ compared to the pacing avoidance algorithm. There was no significant difference in haemodynamics between RVP and ventricular pacing avoidance $(\mathrm{p}=0.055)$. Conclusions HBP provides better acute cardiac function than pacing avoidance algorithms and RVP, in patients with prolonged PR intervals. HBP allows normalisation of prolonged AV delays (unlike pacing avoidance) and does not cause ventricular dyssynchrony (unlike RVP). Clinical trials may be justified to assess whether these acute improvements translate into longer term clinical benefits in patients with bradycardia indications for pacing.
\end{abstract}

\section{Hosted file}

ManuscriptJCE.pdf available at https://authorea.com/users/328624/articles/455808-withinpatient-comparison-of-his-bundle-pacing-right-ventricular-pacing-and-right-ventricularpacing-avoidance-algorithms-in-patients-with-pr-prolongation-acute-haemodynamic-study 